\title{
Viscosity implicit iterative algorithms based on generalized contractions for strictly pseudo-contractive mappings in Banach spaces
}

\author{
Qingqing Cheng ${ }^{a, *}$, Yongfu Su ${ }^{b}$ \\ ${ }^{a}$ Department of Mathematics and LPMC, Nankai University, Tianjin, 300071, China. \\ ${ }^{b}$ Department of Mathematics, Tianjin Polytechnic University, Tianjin, 300387, China. \\ Communicated by M. Eslamian
}

\begin{abstract}
In this manuscript, we construct three viscosity implicit iteration schemes based on generalized contractions for strictly pseudo-contractive mappings. The first scheme is used to approximate a fixed point of a single strictly pseudo-contractive mapping, the second scheme is used to approximate a common fixed point of a finite family of strictly pseudo-contractive mappings, the third scheme is used to approximate a common fixed point of a countable family of strictly pseudo-contractive mappings. Furthermore, three strong convergence theorems based on the purposed iterative schemes are established in the framework of Banach spaces. Finally, three numerical examples are also given to show the efficiency and implementation of our schemes. The main results of this paper modify and improve many important recent results in the literature. (C)2017 All rights reserved.
\end{abstract}

Keywords: Strictly pseudo-contraction, implicit iterative algorithm, viscosity technique, generalized contraction, fixed point. 2010 MSC: 47H05, 47H09, 47H10.

\section{Introduction}

Throughout this paper, we assume that $X$ is a real Banach space and $X^{*}$ is the dual space of $X$. Let $C$ be a subset of $X$. The duality mapping $\mathrm{J}: X \rightarrow 2^{X^{\star}}$ is defined by

$$
\mathrm{J} x=\left\{x^{*} \in \mathrm{X}^{*}:\left\langle x, x^{*}\right\rangle=\|x\|^{2}=\left\|x^{*}\right\|^{2}\right\}, \quad \forall x \in X .
$$

It is well-known that if $X$ is a Hilbert space, then $J$ is the identity mapping and if $X$ is smooth, then $J$ is single-valued, which is denoted by $j$.

Let $T$ be a self-mapping of $C$ and $F(T)$ be the set of fixed points of mapping $T$. That is,

$$
F(T)=\{x \in C: T x=x\} .
$$

Recall that $T: C \rightarrow C$ is said to be L-Lipschitzian if for all $x, y \in C$, there exists a constant $L>0$ such that

$$
\|T x-T y\| \leqslant L\|x-y\| .
$$

If $0<\mathrm{L}<1$, then $\mathrm{T}$ is a contraction and if $\mathrm{L}=1$, then $\mathrm{T}$ is a nonexpansive mapping.

\footnotetext{
*Corresponding author

Email addresses: chengqingqing2006@126.com (Qingqing Cheng), suyongfu@tjpu.edu.cn (Yongfu Su)
} 
$\mathrm{T}: \mathrm{C} \rightarrow \mathrm{C}$ is called to be $\mu$-strictly pseudo-contraction, if there exists a fixed constant $\mu \in(0,1)$ and some $j(x-y) \in J(x-y)$ such that

$$
\langle T x-T y, j(x-y)\rangle \leqslant\|x-y\|^{2}-\mu\|(I-T) x-(I-T) y\|^{2}, \quad \forall x, y \in C,
$$

or equivalently

$$
\langle(I-T) x-(I-T) y, j(x-y)\rangle \geqslant \mu\|(I-T) x-(I-T) y\|^{2}, \quad \forall x, y \in C .
$$

It is well-known that every $\mu$-strictly pseudocontractive mapping is $\frac{1+\mu}{\mu}$-Lipschitzian.

Let $(X, d)$ be a metric space. A mapping $f: X \rightarrow X$ is called a contraction, if there exists a constant $\alpha \in[0,1)$ such that

$$
\|f(x)-f(y)\| \leqslant \alpha\|x-y\|, \quad \forall x, y \in C .
$$

Let $\mathrm{N}$ and $\mathrm{R}^{+}$be the set of all positive integers and all positive real numbers, respectively. A mapping $\psi: R^{+} \rightarrow R^{+}$is said to be an L-function if $\psi(0)=0, \psi(t)>0$ for each $t>0$ and for every $s>0$, there exists $u>s$ such that $\psi(t) \leqslant s$ for each $t \in[s, u]$. As a consequence, every L-function $\psi$ satisfies $\psi(t)<t$ for each $t>0$.

A mapping $f: X \rightarrow X$ is said to be a $(\psi, L)$-contraction if $\psi: R^{+} \rightarrow R^{+}$is said to be an L-function and $d(f(x), f(y))<\psi(d(x, y))$, for all $x, y \in X, x \neq y$.

A mapping $f: X \rightarrow X$ is said to be a Meir-Keeler type mapping if for each $\epsilon>0$ there exists $\delta=\delta(\epsilon)>$ 0 such that for each $x, y \in X$, with $\epsilon \leqslant d(x, y)<\epsilon+\delta$, we have $d(f(x), f(y))<\epsilon$.

Proposition 1.1 ([7]). Let $(\mathrm{X}, \mathrm{d})$ be a metric space and $\mathrm{f}: \mathrm{X} \rightarrow \mathrm{X}$ be a mapping. The following assertions are equivalent:

(i) $\mathrm{f}$ is a Meir-Keeler type mapping;

(ii) there exists an L-function $\psi: \mathrm{R}^{+} \rightarrow \mathrm{R}^{+}$such that $\mathrm{f}$ is a $(\psi, \mathrm{L})$-contraction.

Proposition 1.2 ([15]). Let $\mathrm{C}$ be a convex subset of a Banach space $\mathrm{X}$ and $\mathrm{f}: \mathrm{C} \rightarrow \mathrm{C}$ be a Meir-Keeler type mapping. Then, for each $\epsilon>0$ there exists $r \in(0,1)$ such that

$$
\|x-y\| \geqslant \epsilon \text { implies }\|f(x)-f(y)\| \leqslant r\|x-y\| .
$$

In this paper, generalized contraction mappings refer to Meir-Keeler type mappings or $(\psi, \mathrm{L})$ - contractions and we assume that the L-function from the definition of $(\psi, \mathrm{L})$-contraction is continuous, strictly increasing and $\lim _{t \rightarrow \infty} \eta(t)=\infty$, where $\eta(t)=t-\psi(t)$ for all $t \in R^{+}$.

Fixed point theory plays a very important role for solving all kinds of problems, such as variational inequality problems in Hilbert spaces or Banach spaces, equilibrium problems, optimization problems and so on. A well-known iteration method for approximating fixed points of a nonexpansive mapping is the viscosity approximation method introduced by Moudafi [8] in 2000. Later, the explicit viscosity method for nonexpansive mappings was proposed in the framework of Hilbert spaces by Xu [17] in 2004. For arbitrary $x_{1} \in H$, let $\left\{x_{n}\right\}$ be a sequence in $H$ defined by

$$
x_{n+1}=\alpha_{n} f\left(x_{n}\right)+\left(1-\alpha_{n}\right) T x_{n}, \quad \forall n \in N,
$$

where $f$ and $T$ are contractions and nonexpansive mappings from $\mathrm{H}$ onto itself, respectively. Under certain assumptions imposed on the parameters, the sequence $\left\{x_{n}\right\}$ generated by (1.1) converges strongly to the fixed point $x^{*}$ of $T$ which also solves the following variational inequality:

$$
\left\langle(\mathrm{I}-\mathrm{f}) \mathrm{x}^{*}, x-x^{*}\right\rangle \geqslant 0, \quad \forall x \in \mathrm{F}(\mathrm{T}) .
$$

The implicit midpoint rule is a powerful method for solving ordinary differential equations; see [1, 2, 4, $6,12,18,20$ ] and the references therein. Recently, $\mathrm{Xu}$ et al. [18] considered the following viscosity implicit midpoint rule:

$$
x_{n+1}=\alpha_{n} f\left(x_{n}\right)+\left(1-\alpha_{n}\right) T\left(\frac{x_{n}+x_{n+1}}{2}\right), \quad \forall n \in N
$$


They proved that the iterative sequence defined by (1.3) converges strongly to a fixed point of $T$ which solves the variational inequality (1.2) in Hilbert spaces. In the same year, Ke et al. [6] generalized the viscosity implicit midpoint rule of $\mathrm{Xu}$ et al. [18] to the following two viscosity implicit rules:

$$
x_{n+1}=\alpha_{n} f\left(x_{n}\right)+\left(1-\alpha_{n}\right) T\left(\beta_{n} x_{n}+\left(1-\beta_{n}\right) x_{n+1}\right),
$$

and

$$
x_{n+1}=\alpha_{n} x_{n}+\beta_{n} f\left(x_{n}\right)+\gamma_{n} T\left(\delta_{n} x_{n}+\left(1-\delta_{n}\right) x_{n+1}\right),
$$

where $f$ and $T$ are still contractions and nonexpansive mappings from $H$ onto itself, respectively. They obtained that the sequence $\left\{x_{n}\right\}$ generated by (1.4) and (1.5) converges strongly to a fixed point of nonexpansive mapping $T$, which also solves variational inequality (1.2). In 2016, Yan et al. [19] extended the main results of Ke et al. [6] from Hilbert spaces to uniformly smooth Banach spaces and replaced strict contractions by generalized contractions. They proved that the sequence generated by (1.5) converges strongly to a fixed point $x^{*}$ of nonexpansive mapping $T$, which is also the solution of the following variational inequality in Banach spaces:

$$
\left\langle(I-f) x^{*}, j\left(x-x^{*}\right)\right\rangle \geqslant 0, \quad \forall x \in F(T) .
$$

Very recently, Fan et al. [5] proposed the viscosity approximation method:

$$
x_{n+1}=\alpha_{n} x_{n}+\beta_{n} f\left(x_{n}\right)+\gamma_{n} S_{n} x_{n}
$$

where $S_{n} x=:\left(1-\lambda_{n}\right) x+\lambda_{n} T x, T$ and $f$ be a strictly pseudo-contractive map and contractions, respectively. Under certain assumptions imposed on the parameters, they proved that the sequence $\left\{x_{n}\right\}$ generated by (1.7) converges strongly to the fixed point $x^{*}$ of $T$ which solves the variational inequality (1.6) in uniformly smooth Banach spaces.

Then the following questions naturally arise in connection with above results:

(i) Can we extend the iterative process (1.7) to a general viscosity implicit approximation iterative process?

(ii) Can we replace strict contractions by generalized contractions?

(iii) Can we construct an iterative process for approximating a common fixed point of a finite family of strictly pseudo-contractive mappings?

(iv) Can we construct an iterative process for approximating a common fixed point of a countable family of strictly pseudo-contractive mappings?

The purpose of this paper is to give an affirmative answer to these questions mentioned above. And the structure of this paper is organized as follows: In Section 2, we give some basic definitions and lemmas which will be used in proving our main results. In Section 3, we present three generalized viscosity implicit iteration schemes based on generalized contractions for strictly pseudo-contractive mappings and obtain strong convergence theorems based on the proposed iterative schemes in the framework of Banach spaces. In Section 4, we give three numerical examples to show the efficiency and implementation of our schemes.

\section{Preliminaries}

Let $S(X)=\{x \in X:\|x\|=1\}$. Let the function $\rho_{X}:[0, \infty] \rightarrow[0, \infty]$ be the modulus of smoothness of $X$ defined by

$$
\rho_{X}(t):=\sup \left\{\frac{\|x+y\|+\|x-y\|}{2}-1: x \in S(X),\|y\| \leqslant t\right\} .
$$

A Banach space $X$ is said to be uniformly smooth if $\frac{\rho_{X}(t)}{t} \rightarrow 0$ as $t \rightarrow 0$. A typical example of uniformly smooth Banach spaces is $L^{p}$, where $p>1$. More precisely, $L^{p}$ is $\min \{p, 2\}$-uniformly smooth for every 
$p>1$. Let $q$ be a fixed real number with $1<q \leqslant 2$. Then a Banach space $X$ is said to be $q$-uniformly smooth if there exists a constant $c>0$ such that $x(t) \leqslant c t^{q}$ for all $t>0$. In a smooth Banach space, $J$ is single-valued. In a uniformly smooth Banach space, J is uniformly continuous on bounded subsets.

To obtain the result of this paper, we also need the following lemmas and definition.

Lemma 2.1 ([9]). Let X be a real smooth Banach space. Suppose one of the followings holds:

(i) $j$ is uniformly continuous on any bounded subset of $X$.

(ii) $\langle x-y, j(x)-j(y)\rangle \leqslant\|x-y\|^{2}$, for all $x, y \in X$.

(iii) For any bounded subset $\mathrm{D}$ of $\mathrm{X}$, there is a $\mathrm{c}$ such that

$$
\langle x-y, j(x)-j(y)\rangle \leqslant c(\|x-y\|), \quad \forall x, y \in D,
$$

where c satisfies $\lim _{\mathrm{t} \rightarrow 0^{+}} \frac{\mathrm{c}(\mathrm{t})}{\mathrm{t}}=0$.

Then, for any $\varepsilon>0$ and any bounded subset $\mathrm{C}$ there is $\delta>0$ such that

$$
\|t x+(1-t) y\|^{2} \leqslant 2 t\langle x, j y\rangle+2 t \varepsilon+(1-2 t)\|y\|^{2}
$$

for any $x, y \in C$ and $\mathrm{t} \in[0, \delta)$.

Definition 2.2 ([10]). Let $C$ be a nonempty subset of a Banach space $X$ and $\left\{T_{n}\right\}$ be a sequence of mappings from $C$ into $X$ with $\bigcap_{n=1}^{\infty} F\left(T_{n}\right) \neq \emptyset$. For any bounded subset $B$ of $C$, we say that $\left\{T_{n}\right\}$ satisfies the PUcondition, if there exists a continuous and increasing function $h_{B}: R^{+} \rightarrow R^{+}$, and for all $k, l \in N$ such that

$$
h_{B}(0)=0, \quad \lim _{k, l \rightarrow \infty} \sup _{\omega \in B} h_{B}\left(\left\|T_{k} \omega-T_{l} \omega\right\|\right)=0 .
$$

Lemma 2.3 ([10]). Let $\left\{T_{n}\right\}$ be a sequence of mappings from $C$ into $X$. Suppose that for any bounded subset $B$ of $C$, there exists a continuous and increasing function $\mathrm{h}_{\mathrm{B}}: \mathrm{R}^{+} \rightarrow \mathrm{R}^{+}$satisfying (2.1). Then the following hold:

(i) For each $\mathrm{x} \in \mathrm{C},\left\{\mathrm{T}_{\mathrm{n}}\right\}$ converges strongly to some point of $\mathrm{C}$.

(ii) If the mapping $\mathrm{T}: \mathrm{C} \rightarrow \mathrm{X}$ be defined by $\mathrm{T} x=\lim _{\mathrm{n} \rightarrow \infty} \mathrm{T}_{\mathrm{n}} \mathrm{x}$, for all $\mathrm{x} \in \mathrm{C}$, then $\lim _{\mathrm{n} \rightarrow \infty} \sup _{\boldsymbol{\omega} \in \mathrm{B}} \mathrm{h}_{\mathrm{B}}(\| \mathrm{T} \omega-$ $\left.\mathrm{T}_{\mathrm{n}} \omega \|\right)=0$. Moreover, the properties of $\mathrm{h}_{\mathrm{B}}$ imply that

$$
\lim _{n \rightarrow \infty} \sup _{\omega \in B}\left\|T \omega-T_{n} \omega\right\|=0 .
$$

Lemma 2.4 ([14]). Let $\left\{x_{n}\right\}$ and $\left\{z_{n}\right\}$ be bounded sequences in a Banach space $X$ and let $\left\{\tau_{n}\right\}$ be a sequence in $[0,1]$ with $0<\liminf _{n \rightarrow \infty} \tau_{n} \leqslant \limsup _{n \rightarrow \infty} \tau_{n}<1$. Suppose $x_{n+1}=\tau_{n} z_{n}+\left(1-\tau_{n}\right) x_{n}$ for all integers $n \geqslant 0$ and $\limsup _{n \rightarrow \infty}\left(\left\|z_{n+1}-z_{n}\right\|-\left\|x_{n+1}-x_{n}\right\|\right) \leqslant 0$. Then, $\lim _{n \rightarrow \infty}\left\|z_{n}-x_{n}\right\|=0$.

Lemma 2.5 ([17]). Assume that $\left\{a_{n}\right\}$ is a sequence of nonnegative real numbers such that

$$
a_{n+1} \leqslant\left(1-b_{n}\right) a_{n}+c_{n}
$$

where $b_{n}$ is a sequence in $(0,1)$ and $\left\{c_{n}\right\}$ is a sequence such that

(i) $\sum_{n=1}^{\infty} b_{n}=\infty$;

(ii) $\limsup _{n \rightarrow \infty} \frac{\mathfrak{c}_{n}}{b_{n}} \leqslant 0$ or $\sum_{n=1}^{\infty}\left|c_{n}\right|<\infty$.

Then $\lim _{n \rightarrow \infty} a_{n}=0$.

Let $C$ and $K$ be subsets of a Banach space $X$. A mapping $P$ from $C$ into $K$ is called sunny [3] if $P(P x+t(x-P x))=P x$ for $x \in C$ with $P x+t(x-P x) \in C$ and $t \geqslant 0$. The following is proved in [11]; see also [16]. 
Lemma 2.6 ([11]). Let $\mathrm{E}$ be a smooth Banach space and let $\mathrm{J}$ be the duality mapping from $\mathrm{E}$ into $\mathrm{E}^{*}$, that is, $\langle\mathrm{x}, \mathrm{J}(\mathrm{x})\rangle=\|\mathrm{x}\|^{2}=\|\mathrm{J}(\mathrm{x})\|^{2}$ for all $\mathrm{x} \in \mathrm{E}$. Let $\mathrm{C}$ be a convex subset of $\mathrm{E}$, let $\mathrm{K}$ be a subset of $\mathrm{C}$ and let $\mathrm{P}$ be $a$ retraction from $\mathrm{C}$ onto $\mathrm{K}$. Then the following are equivalent:

(i) $\langle x-P x, J(P x-y)\rangle \geqslant 0$ for all $x \in C$ and $y \in K$;

(ii) $\mathrm{P}$ is both sunny and nonexpansive.

Hence, there is at most one sunny nonexpansive retraction from $C$ onto $K$. Note that if $E$ is a Hilbert space and $\mathrm{K}$ is closed and convex, then the metric projection and the sunny nonexpansive retraction from $\mathrm{C}$ onto $\mathrm{K}$ coincide.

Lemma 2.7 ([15]). Let $\mathrm{C}$ be a closed convex subset of a smooth Banach space $\mathrm{E}$. Let $\mathrm{K}$ be a subset of $\mathrm{C}$ and let $\mathrm{P}$ be the unique sunny nonexpansive retraction from $\mathrm{C}$ onto $\mathrm{K}$. Let $\Phi$ be a mapping on $\mathrm{C}$, and let $\mathrm{z}$ belong to $\mathrm{K}$. Then the following are equivalent:

(i) $z$ is a fixed point of $\mathrm{P} \circ \Phi$;

(ii) $z$ is a solution of a variational inequality $\langle\Phi z-z, J(z-y)\rangle \geqslant 0$ for all $y \in K$.

Lemma 2.8 ([13]). Let $\mathrm{C}$ be a nonempty closed and convex subset of a uniformly smooth Banach space X. Let $\mathrm{T}: \mathrm{C} \rightarrow \mathrm{C}$ be a nonexpansive mapping such that $\mathrm{F}(\mathrm{T}) \neq \emptyset$ and $\mathrm{f}: \mathrm{C} \rightarrow \mathrm{C}$ be a generalized contraction mapping. Then $\left\{x_{t}\right\}$ defined by $x_{t}=\operatorname{tf}\left(x_{t}\right)+(1-t) T x_{t}$ for $t \in(0,1)$, converges strongly to $\hat{x} \in \mathrm{F}(T)$, which solves the variational inequality:

$$
\langle f(\hat{x})-\hat{x}, j(z-\hat{x})\rangle \leqslant 0, \quad \forall z \in F(T) .
$$

From Lemma 2.7, we know that, let $P$ be the unique sunny nonexpansive retraction from $C$ onto $F(T)$, the variational inequality (2.2) is equivalent to $P \circ f(z)=z$, that is, $z$ is a fixed point of $P \circ f$.

Lemma 2.9 ([13]). Let $C$ be a nonempty closed and convex subset of a uniformly smooth Banach space X. Let $\mathrm{T}: \mathrm{C} \rightarrow \mathrm{C}$ be a nonexpansive mapping such that $\mathrm{F}(\mathrm{T}) \neq \emptyset$ and $\mathrm{f}: \mathrm{C} \rightarrow \mathrm{C}$ be a generalized contraction mapping. Assume that $\left\{\mathrm{x}_{\mathrm{t}}\right\}$ defined by $\mathrm{x}_{\mathrm{t}}=\mathrm{tf}\left(\mathrm{x}_{\mathrm{t}}\right)+(1-\mathrm{t}) \mathrm{T} \mathrm{x}_{\mathrm{t}}$ for $\mathrm{t} \in(0,1)$, converges strongly to $\hat{\mathrm{x}} \in \mathrm{F}(\mathrm{T})$ as $\mathrm{t} \rightarrow 0$. Suppose that $\left\{x_{n}\right\}$ is a bounded sequence such that $x_{n}-T x_{n} \rightarrow 0$ as $n \rightarrow \infty$. Then

$$
\limsup _{n \rightarrow \infty}\left\langle f(\hat{x})-\hat{x}, j\left(x_{n}-\hat{x}\right)\right\rangle \leqslant 0 .
$$

\section{Main results}

Theorem 3.1. Let $\mathrm{X}$ be a real uniformly smooth Banach space and $\mathrm{C}$ be a nonempty bounded closed convex subset of $\mathrm{X}$. Let $\mathrm{T}: \mathrm{C} \rightarrow \mathrm{C}$ be a $\mu$-strictly pseudo-contractive mapping such that $\mathrm{F}(\mathrm{T}) \neq \emptyset$ and $\mathrm{f}: \mathrm{C} \rightarrow \mathrm{C}$ a generalized contraction mapping. Pick any $\mathrm{x}_{0} \in \mathrm{C}$. Let $\left\{\mathrm{x}_{\mathrm{n}}\right\}$ be a sequence generated by the following algorithm

$$
x_{n+1}=\alpha_{n} x_{n}+\beta_{n} f\left(x_{n}\right)+\gamma_{n} S_{n}\left(\delta_{n} x_{n}+\left(1-\delta_{n}\right) x_{n+1}\right),
$$

where $S_{n} x=:\left(1-\lambda_{n}\right) x+\lambda_{n} T x$ and assume that $\left\{\alpha_{n}\right\},\left\{\beta_{n}\right\},\left\{\gamma_{n}\right\},\left\{\delta_{n}\right\}$ and $\left\{\lambda_{n}\right\}$ are five sequences in $[0,1]$ satisfying the following conditions:

(i) $\alpha_{n}+\beta_{n}+\gamma_{n}=1$;

(ii) $\lim _{n \rightarrow \infty}\left|\alpha_{n+1}-\alpha_{n}\right|=0$ and $0<\liminf _{n \rightarrow \infty} \alpha_{n} \leqslant \limsup _{n \rightarrow \infty} \alpha_{n}<1$;

(iii) $\sum_{n=0}^{\infty} \beta_{n}=\infty, \lim _{n \rightarrow \infty} \beta_{n}=0$;

(iv) $0<\delta_{\mathfrak{n}} \leqslant \delta_{n+1}<1$;

(v) $\lim _{n \rightarrow \infty}\left|\lambda_{n+1}-\lambda_{n}\right|=0$.

Then sequence $\left\{x_{n}\right\}$ converges strongly to a fixed point $\hat{x}$ of $\mathrm{T}$, which is also the solution of the variational inequality

$$
\langle(I-f) \hat{x}, j(x-\hat{x})\rangle \geqslant 0, \quad \forall x \in F(T) .
$$

Moreover, assume that $\mathrm{P}$ is the unique sunny nonexpansive retraction from $\mathrm{C}$ onto $\mathrm{F}(\mathrm{T})$, the variational inequality (3.2) is equivalent to $\mathrm{P} \circ \mathrm{f}(\hat{\mathrm{x}})=\hat{\mathrm{x}}$. 
Proof. First, we show that $S_{n}$ is nonexpansive, for all $n \geqslant 0$. Indeed, for all $x, y \in C$, taking

$$
0<\varepsilon \leqslant \mu\|\mathrm{T} x-\mathrm{T} y-(x-y)\|^{2},
$$

by Lemma 2.1, we have

$$
\begin{aligned}
\left\|S_{n} x-S_{n} y\right\|^{2}= & \left\|\left(1-\lambda_{n}\right) x+\lambda_{n} T x-\left(1-\lambda_{n}\right) y-\lambda_{n} T y\right\|^{2} \\
= & \left\|\left(1-\lambda_{n}\right)(x-y)+\lambda_{n}(T x-T y)\right\|^{2} \\
\leqslant & 2 \lambda_{n}\langle T x-T y, j(x-y)\rangle+2 \varepsilon \lambda_{n}+\left(1-2 \lambda_{n}\right)\|x-y\|^{2} \\
\leqslant & 2 \lambda_{n}\left(\|x-y\|^{2}-\mu\|T x-T y-(x-y)\|^{2}\right) \\
& +\left(1-2 \lambda_{n}\right)\|x-y\|^{2}+2 \varepsilon \lambda_{n} \\
\leqslant & \|x-y\|^{2}-2 \lambda_{n} \mu\|T x-T y-(x-y)\|^{2}+2 \varepsilon \lambda_{n} \\
\leqslant & \|x-y\|^{2} .
\end{aligned}
$$

It is obvious that for each $n \in N, x \in F\left(S_{n}\right) \Leftrightarrow x \in F(T)$. So, $F\left(S_{n}\right)=F(T) \neq \emptyset$.

Next, we show that $\lim _{n \rightarrow \infty}\left\|x_{n+1}-x_{n}\right\|=0$. Set $z_{n}=\frac{x_{n+1}-\alpha_{n} x_{n}}{1-\alpha_{n}}, y_{n}=\delta_{n} x_{n}+\left(1-\delta_{n}\right) x_{n+1}$. We obtain that

$$
\begin{aligned}
\left\|z_{n+1}-z_{n}\right\|= & \left\|\frac{x_{n+2}-\alpha_{n+1} x_{n+1}}{1-\alpha_{n+1}}-\frac{x_{n+1}-\alpha_{n} x_{n}}{1-\alpha_{n}}\right\| \\
= & \left\|\frac{\beta_{n+1} f\left(x_{n+1}\right)+\gamma_{n+1} S_{n+1} y_{n+1}}{1-\alpha_{n+1}}-\frac{\beta_{n} f\left(x_{n}\right)+\gamma_{n} S_{n} y_{n}}{1-\alpha_{n}}\right\| \\
= & \| \frac{\beta_{n+1}}{1-\alpha_{n+1}}\left(f\left(x_{n+1}\right)-f\left(x_{n}\right)\right)+\frac{\gamma_{n+1}}{1-\alpha_{n+1}}\left(S_{n+1} y_{n+1}-S_{n} y_{n}\right) \\
& +\left(\frac{\beta_{n+1}}{1-\alpha_{n+1}}-\frac{\beta_{n}}{1-\alpha_{n}}\right) f\left(x_{n}\right)+\left(\frac{\gamma_{n+1}}{1-\alpha_{n+1}}-\frac{\gamma_{n}}{1-\alpha_{n}}\right) S_{n} y_{n} \| \\
= & \| \frac{\beta_{n+1}}{1-\alpha_{n+1}}\left(f\left(x_{n+1}\right)-f\left(x_{n}\right)\right)+\frac{\gamma_{n+1}}{1-\alpha_{n+1}}\left(S_{n+1} y_{n+1}-S_{n} y_{n}\right) \\
& +\left(\frac{\beta_{n+1}}{1-\alpha_{n+1}}-\frac{\beta_{n}}{1-\alpha_{n}}\right)\left[f\left(x_{n}\right)-S_{n} y_{n}\right] \| \\
\leqslant & \frac{\beta_{n+1}}{1-\alpha_{n+1}} \psi\left(\left\|x_{n+1}-x_{n}\right\|\right)+\frac{\gamma_{n+1}}{1-\alpha_{n+1}}\left\|S_{n+1} y_{n+1}-S_{n} y_{n}\right\| \\
& +\mid \frac{\beta_{n+1}}{1-\alpha_{n+1}}-\frac{\beta_{n}}{1-\alpha_{n}}\left\|f\left(x_{n}\right)-S_{n} y_{n}\right\| .
\end{aligned}
$$

Since $C$ is a nonempty bounded closed convex subset of $X$, then $\left\{x_{n}\right\},\left\{y_{n}\right\}$ and $\left\{T y_{n}\right\}$ are bounded. Hence there exists $M=\sup _{n \geqslant 0}\left\{\left\|T y_{n}-y_{n}\right\|\right\}$. Again from (3.1) and the property of $S_{n}$, we have

$$
\begin{aligned}
\left\|S_{n+1} y_{n+1}-S_{n} y_{n}\right\|= & \left\|S_{n+1} y_{n+1}-S_{n+1} y_{n}+S_{n+1} y_{n}-S_{n} y_{n}\right\| \\
\leqslant & \left\|y_{n+1}-y_{n}\right\|+\|\left(1-\lambda_{n+1}\right) y_{n}+\lambda_{n+1} T y_{n} \\
& -\left(1-\lambda_{n}\right) y_{n}-\lambda_{n} T y_{n} \| \\
= & \left\|y_{n+1}-y_{n}\right\|+\left|\lambda_{n+1}-\lambda_{n}\right|\left\|T y_{n}-y_{n}\right\| \\
\leqslant & \left\|y_{n+1}-y_{n}\right\|+M\left|\lambda_{n+1}-\lambda_{n}\right| .
\end{aligned}
$$

Also,

$$
\begin{aligned}
\left\|y_{n+1}-y_{n}\right\| & =\left\|\delta_{n+1} x_{n+1}+\left(1-\delta_{n+1}\right) x_{n+2}-\delta_{n} x_{n}-\left(1-\delta_{n}\right) x_{n+1}\right\| \\
& =\left\|\left(1-\delta_{n+1}\right)\left(x_{n+2}-x_{n+1}\right)+\delta_{n}\left(x_{n+1}-x_{n}\right)\right\| \\
& \leqslant\left(1-\delta_{n+1}\right)\left\|x_{n+2}-x_{n+1}\right\|+\delta_{n}\left\|x_{n+1}-x_{n}\right\|,
\end{aligned}
$$


and

$$
\begin{aligned}
\left\|x_{n+2}-x_{n+1}\right\|= & \| \alpha_{n+1} x_{n+1}+\beta_{n+1} f\left(x_{n+1}\right)+\gamma_{n+1} S_{n+1} y_{n+1} \\
& -\alpha_{n} x_{n}-\beta_{n} f\left(x_{n}\right)-\gamma_{n} S_{n} y_{n} \| \\
= & \| \alpha_{n+1}\left(x_{n+1}-x_{n}\right)+\beta_{n+1}\left[f\left(x_{n+1}\right)-f\left(x_{n}\right)\right] \\
& +\gamma_{n+1}\left(S_{n+1} y_{n+1}-S_{n} y_{n}\right)+\left(\alpha_{n+1}-\alpha_{n}\right) x_{n} \\
& +\left(\beta_{n+1}-\beta_{n}\right) f\left(x_{n}\right)+\left(\gamma_{n+1}-\gamma_{n}\right) S_{n} y_{n} \| \\
= & \| \alpha_{n+1}\left(x_{n+1}-x_{n}\right)+\beta_{n+1}\left[f\left(x_{n+1}\right)-f\left(x_{n}\right)\right] \\
& +\gamma_{n+1}\left(S_{n+1} y_{n+1}-S_{n} y_{n}\right)+\left(\alpha_{n+1}-\alpha_{n}\right)\left(x_{n}-S_{n} y_{n}\right) \\
& +\left(\beta_{n+1}-\beta_{n}\right)\left(f\left(x_{n}\right)-S_{n} y_{n}\right) \| \\
\leqslant & \alpha_{n+1}\left\|x_{n+1}-x_{n}\right\|+\beta_{n+1} \psi\left(\left\|x_{n+1}-x_{n}\right\|\right) \\
& +\left|\alpha_{n+1}-\alpha_{n}\right|\left\|x_{n}-S_{n} y_{n}\right\|+\left|\beta_{n+1}-\beta_{n}\right|\left\|f\left(x_{n}\right)-S_{n} y_{n}\right\| \\
& +\gamma_{n+1}\left\|S_{n+1} y_{n+1}-S_{n} y_{n}\right\| .
\end{aligned}
$$

Substituting (3.4) and (3.5) into (3.6), we obtain that

$$
\begin{aligned}
\left\|x_{n+2}-x_{n+1}\right\| \leqslant & \frac{\alpha_{n+1}+\gamma_{n+1} \delta_{n}+\beta_{n+1} \psi}{1-\gamma_{n+1}\left(1-\delta_{n+1}\right)}\left\|x_{n+1}-x_{n}\right\|+\frac{\left|\alpha_{n+1}-\alpha_{n}\right|}{1-\gamma_{n+1}\left(1-\delta_{n+1}\right)}\left\|x_{n}-S_{n} y_{n}\right\| \\
& +\frac{\left|\beta_{n+1}-\beta_{n}\right|}{1-\gamma_{n+1}\left(1-\delta_{n+1}\right)}\left\|f\left(x_{n}\right)-S_{n} y_{n}\right\|+\frac{\gamma_{n+1} M}{1-\gamma_{n+1}\left(1-\delta_{n+1}\right)}\left\|\lambda_{n+1}-\lambda_{n}\right\| .
\end{aligned}
$$

Substituting (3.7), (3.5) and (3.4) into (3.3), we obtain that

$$
\begin{aligned}
& \left\|z_{n+1}-z_{n}\right\| \leqslant\left[\frac{\beta_{n+1} \psi}{1-\alpha_{n+1}}+\frac{\gamma_{n+1}\left(1-\delta_{n+1}\right)}{1-\alpha_{n+1}} \times \frac{\alpha_{n+1}+\gamma_{n+1} \delta_{n}+\beta_{n+1} \psi}{1-\gamma_{n+1}\left(1-\delta_{n+1}\right)}+\frac{\gamma_{n+1} \delta_{n}}{1-\alpha_{n+1}}\right]\left\|x_{n+1}-x_{n}\right\| \\
& +\frac{\gamma_{n+1}\left(1-\delta_{n+1}\right)}{1-\alpha_{n+1}} \times \frac{\left|\alpha_{n+1}-\alpha_{n}\right|}{1-\gamma_{n+1}\left(1-\delta_{n+1}\right)}\left\|x_{n}-S_{n} y_{n}\right\| \\
& +\left[\frac{\gamma_{n+1}\left(1-\delta_{n+1}\right)}{1-\alpha_{n+1}} \times \frac{\left|\beta_{n+1}-\beta_{n}\right|}{1-\gamma_{n+1}\left(1-\delta_{n+1}\right)}+\left|\frac{\beta_{n+1}}{1-\alpha_{n+1}}-\frac{\beta_{n}}{1-\alpha_{n}}\right|\right]\left\|f\left(x_{n}\right)-S_{n} y_{n}\right\| \\
& +\left[\frac{\gamma_{n+1}\left(1-\delta_{n+1}\right)}{1-\alpha_{n+1}} \times \frac{\gamma_{n+1} M}{1-\gamma_{n+1}\left(1-\delta_{n+1}\right)}+\frac{\gamma_{n+1} M}{1-\alpha_{n+1}}\right]\left\|\lambda_{n+1}-\lambda_{n}\right\| \\
& \leqslant \frac{\gamma_{n+1} \delta_{n}+\beta_{n+1} \psi+\gamma_{n+1}\left(1-\delta_{n+1}\right) \alpha_{n+1}}{\left[1-\gamma_{n+1}\left(1-\delta_{n+1}\right)\right]\left(1-\alpha_{n+1}\right)}\left\|x_{n+1}-x_{n}\right\| \\
& +\frac{\left|\alpha_{n+1}-\alpha_{n}\right|}{1-\gamma_{n+1}\left(1-\delta_{n+1}\right)}\left\|x_{n}-S_{n} y_{n}\right\|+\left[\frac{\left|\beta_{n+1}-\beta_{n}\right|}{1-\gamma_{n+1}\left(1-\delta_{n+1}\right)}+\left|\frac{\beta_{n+1}}{1-\alpha_{n+1}}-\frac{\beta_{n}}{1-\alpha_{n}}\right|\right] \\
& \left\|f\left(x_{n}\right)-S_{n} y_{n}\right\|+\left[\frac{\gamma_{n+1} M}{1-\gamma_{n+1}\left(1-\delta_{n+1}\right)}+\frac{\gamma_{n+1} M}{1-\alpha_{n+1}}\right]\left\|\lambda_{n+1}-\lambda_{n}\right\| \\
& =\left(1-\frac{\beta_{n+1} \eta+\gamma_{n+1}\left(\delta_{n+1}-\delta_{n}\right)}{\left[1-\gamma_{n+1}\left(1-\delta_{n+1}\right)\right]\left(1-\alpha_{n+1}\right)}\right)\left\|x_{n+1}-x_{n}\right\| \\
& +\frac{\left|\alpha_{n+1}-\alpha_{n}\right|}{1-\gamma_{n+1}\left(1-\delta_{n+1}\right)}\left\|x_{n}-S_{n} y_{n}\right\|+\left[\frac{\left|\beta_{n+1}-\beta_{n}\right|}{1-\gamma_{n+1}\left(1-\delta_{n+1}\right)}+\left|\frac{\beta_{n+1}}{1-\alpha_{n+1}}-\frac{\beta_{n}}{1-\alpha_{n}}\right|\right] \\
& \left\|f\left(x_{n}\right)-S_{n} y_{n}\right\|+\left[\frac{\gamma_{n+1} M}{1-\gamma_{n+1}\left(1-\delta_{n+1}\right)}+\frac{\gamma_{n+1} M}{1-\alpha_{n+1}}\right]\left\|\lambda_{n+1}-\lambda_{n}\right\| \\
& \leqslant\left(1-\frac{\beta_{n+1} \eta}{1-\alpha_{n+1}}\right)\left\|x_{n+1}-x_{n}\right\|+\frac{\left|\alpha_{n+1}-\alpha_{n}\right|}{1-\gamma_{n+1}\left(1-\delta_{n+1}\right)}\left\|x_{n}-S_{n} y_{n}\right\| \\
& +\left[\frac{\left|\beta_{n+1}-\beta_{n}\right|}{1-\gamma_{n+1}\left(1-\delta_{n+1}\right)}+\left|\frac{\beta_{n+1}}{1-\alpha_{n+1}}-\frac{\beta_{n}}{1-\alpha_{n}}\right|\right]\left\|f\left(x_{n}\right)-S_{n} y_{n}\right\| \\
& +\left[\frac{\gamma_{n+1} M}{1-\gamma_{n+1}\left(1-\delta_{n+1}\right)}+\frac{\gamma_{n+1} M}{1-\alpha_{n+1}}\right]\left\|\lambda_{n+1}-\lambda_{n}\right\| \text {. }
\end{aligned}
$$


Since $C$ is a nonempty bounded closed convex subset of $X$, then $\left\{x_{n}\right\},\left\{y_{n}\right\},\left\{f\left(x_{n}\right)\right\}$ and $\left\{S_{n} y_{n}\right\}$ are bounded. Hence $\left\|x_{n}-S_{n} y_{n}\right\|$ and $\left\|f\left(x_{n}\right)-S_{n} y_{n}\right\|$ are bounded. Again from conditions (ii), (iii) and (v), we have

$$
\limsup _{n \rightarrow \infty}\left(\left\|z_{n+1}-z_{n}\right\|-\left\|x_{n+1}-x_{n}\right\|\right) \leqslant 0 .
$$

By using Lemma 2.4, we have

$$
\lim _{n \rightarrow \infty}\left\|z_{n}-x_{n}\right\|=0
$$

Therefore,

$$
\lim _{n \rightarrow \infty}\left\|x_{n+1}-x_{n}\right\|=\lim _{n \rightarrow \infty}\left(1-\alpha_{n}\right)\left\|z_{n}-x_{n}\right\|=0 .
$$

Next, we prove that $\lim _{n \rightarrow \infty}\left\|x_{n}-S_{n} x_{n}\right\|=0$. Since

$$
\left\|x_{n}-S_{n} x_{n}\right\| \leqslant\left\|x_{n}-x_{n+1}\right\|+\left\|x_{n+1}-S_{n} x_{n}\right\|,
$$

also,

$$
\begin{aligned}
\left\|x_{n+1}-S_{n} x_{n}\right\|= & \left\|\alpha_{n} x_{n}+\beta_{n} f\left(x_{n}\right)+\gamma_{n} S_{n}\left(\delta_{n} x_{n}+\left(1-\delta_{n}\right) x_{n+1}\right)-S_{n} x_{n}\right\| \\
= & \| \alpha_{n}\left(x_{n}-S_{n} x_{n}\right)+\beta_{n}\left(f\left(x_{n}\right)-S_{n} x_{n}\right) \\
& +\gamma_{n}\left[S_{n}\left(\delta_{n} x_{n}+\left(1-\delta_{n}\right) x_{n+1}\right)-S_{n} x_{n}\right] \| \\
\leqslant & \alpha_{n}\left\|x_{n}-S_{n} x_{n}\right\|+\beta_{n}\left\|f\left(x_{n}\right)-S_{n} x_{n}\right\| \\
& +\gamma_{n}\left(1-\delta_{n}\right)\left\|x_{n+1}-x_{n}\right\| .
\end{aligned}
$$

Substituting (3.9) into (3.8), we obtain that

$$
\left\|x_{n}-S_{n} x_{n}\right\| \leqslant \frac{\beta_{n}}{1-\alpha_{n}}\left\|f\left(x_{n}\right)-S_{n} x_{n}\right\|+\frac{1+\gamma_{n}\left(1-\delta_{n}\right)}{1-\alpha_{n}}\left\|x_{n+1}-x_{n}\right\|
$$

From conditions (ii), (iii) and $\lim _{n \rightarrow \infty}\left\|x_{n+1}-x_{n}\right\|=0$, we have

$$
\lim _{n \rightarrow \infty}\left\|x_{n}-S_{n} x_{n}\right\|=0
$$

Let $\left\{x_{t}\right\}$ be a sequence defined by $x_{t}=\operatorname{tf}\left(x_{t}\right)+(1-t) S_{n} x_{t}$ for $t \in(0,1)$, from Lemma 2.8, $\left\{x_{t}\right\}$ converges strongly to $\hat{x} \in F\left(S_{n}\right)=F(T)$, which solves the variational inequality:

$$
\langle f(\hat{x})-\hat{x}, j(x-\hat{x})\rangle \leqslant 0, \quad \forall x \in F(T),
$$

that is,

$$
\langle(I-f) \hat{x}, j(x-\hat{x})\rangle \geqslant 0, \quad \forall x \in F(T) .
$$

Moreover, from Lemma 2.7, we have $P \circ f(\hat{x})=\hat{x}$, where $P$ is the unique sunny nonexpansive retraction from $C$ onto $F(T)$. Again since $\left\{x_{n}\right\}$ is bounded and $\lim _{n \rightarrow \infty}\left\|x_{n}-S_{n} x_{n}\right\|=0$, by Lemma 2.9, we have

$$
\limsup _{n \rightarrow \infty}\left\langle f(\hat{x})-\hat{x}, j\left(x_{n}-\hat{x}\right)\right\rangle \leqslant 0 .
$$

Finally, we show that $x_{n} \rightarrow \hat{x}$ as $n \rightarrow \infty$.

Assume that the sequence $\left\{x_{n}\right\}$ does not converge strongly to $\hat{x} \in F(T)$. Then there exist $\epsilon>0$ and a subsequence $\left\{x_{n_{i}}\right\}$ of $\left\{x_{n}\right\}$ such that $\left\|x_{n_{i}}-\hat{x}\right\| \geqslant \epsilon$, for all $i \geqslant 0$. From Proposition 1.2, for this $\epsilon$ there exists $r \in(0,1)$ such that

$$
\left\|f\left(x_{n_{i}}\right)-f(\hat{x})\right\| \leqslant r\left\|x_{n_{i}}-\hat{x}\right\| .
$$


Then we have

$$
\begin{aligned}
\left\|x_{n_{i}+1}-\hat{x}\right\|^{2}= & \left\langle\alpha_{n_{i}} x_{n_{i}}+\beta_{n_{i}} f\left(x_{n_{i}}\right)+\gamma_{n_{i}} S_{n_{i}}\left(y_{n_{i}}\right)-\hat{x}, j\left(x_{n_{i}+1}-\hat{x}\right)\right\rangle \\
= & \alpha_{n_{i}}\left\langle x_{n_{i}}-\hat{x}, j\left(x_{n_{i}+1}-\hat{x}\right)\right\rangle+\beta_{n_{i}}\left\langle f\left(x_{n_{i}}\right)-\hat{x}, j\left(x_{n_{i}+1}-\hat{x}\right)\right\rangle \\
& +\gamma_{n_{i}}\left\langle S_{n_{i}}\left(y_{n_{i}}\right)-\hat{x}, j\left(x_{n_{i}+1}-\hat{x}\right)\right\rangle \\
= & \alpha_{n_{i}}\left\langle x_{n_{i}}-\hat{x}, j\left(x_{n_{i}+1}-\hat{x}\right)\right\rangle+\beta_{n_{i}}\left\langle f\left(x_{n_{i}}\right)-f(\hat{x}), j\left(x_{n_{i}+1}-\hat{x}\right)\right\rangle \\
& +\beta_{n_{i}}\left\langle f(\hat{x})-\hat{x}, j\left(x_{n_{i}+1}-\hat{x}\right)\right\rangle+\gamma_{n_{i}}\left\langle S_{n_{i}}\left(y_{n_{i}}\right)-\hat{x}, j\left(x_{n_{i}+1}-\hat{x}\right)\right\rangle \\
\leqslant & \alpha_{n_{i}}\left\|x_{n_{i}}-\hat{x}\right\|\left\|x_{n_{i}+1}-\hat{x}\right\|+\beta_{n_{i}} r\left\|x_{n_{i}}-\hat{x}\right\|\left\|x_{n_{i}+1}-\hat{x}\right\| \\
& +\gamma_{n_{i}}\left\|S_{n_{i}}\left(y_{n_{i}}\right)-\hat{x}\right\|\left\|x_{n_{i}+1}-\hat{x}\right\|+\beta_{n_{i}}\left\langle f(\hat{x})-\hat{x}, j\left(x_{n_{i}+1}-\hat{x}\right)\right\rangle .
\end{aligned}
$$

Since

$$
\begin{aligned}
\left\|S_{n_{i}}\left(y_{n_{i}}\right)-\hat{x}\right\| & =\left\|S_{n_{i}}\left(y_{n_{i}}\right)-S_{n_{i}} \hat{x}\right\| \\
& \leqslant\left\|y_{n_{i}}-\hat{x}\right\| \\
& =\left\|\delta_{n_{i}} x_{n_{i}}+\left(1-\delta_{n_{i}}\right) x_{n_{i}+1}-\hat{x}\right\| \\
& \leqslant \delta_{n_{i}}\left\|x_{n_{i}}-\hat{x}\right\|+\left\|\left(1-\delta_{n_{i}}\right) x_{n_{i}+1}-\hat{x}\right\| .
\end{aligned}
$$

Substituting (3.12) into (3.11), we obtain that

$$
\begin{aligned}
\left\|x_{n_{i}+1}-\hat{x}\right\|^{2} \leqslant & \left(\alpha_{n_{i}}+\beta_{n_{i}} r+\gamma_{n_{i}} \delta_{n_{i}}\right)\left\|x_{n_{i}}-\hat{x}\right\|\left\|x_{n_{i}+1}-\hat{x}\right\| \\
& +\gamma_{n_{i}}\left(1-\delta_{n_{i}}\right)\left\|x_{n_{i}+1}-\hat{x}\right\|^{2}+\beta_{n_{i}}\left\langle f(\hat{x})-\hat{x}, j\left(x_{n_{i}+1}-\hat{x}\right)\right\rangle \\
\leqslant & \frac{\alpha_{n_{i}}+\beta_{n_{i}} r+\gamma_{n_{i}} \delta_{n_{i}}}{2}\left\|x_{n_{i}}-\hat{x}\right\|^{2}+\frac{\alpha_{n_{i}}+\beta_{n_{i}} r+\gamma_{n_{i}} \delta_{n_{i}}}{2}\left\|x_{n_{i}+1}-\hat{x}\right\|^{2} \\
& +\gamma_{n_{i}}\left(1-\delta_{n_{i}}\right)\left\|x_{n_{i}+1}-\hat{x}\right\|^{2}+\beta_{n_{i}}\left\langle f(\hat{x})-\hat{x}, j\left(x_{n_{i}+1}-\hat{x}\right)\right\rangle .
\end{aligned}
$$

Hence,

$$
\begin{aligned}
\left\|x_{n_{i}+1}-\hat{x}\right\|^{2} \leqslant & \frac{\alpha_{n_{i}}+\beta_{n_{i}} r+\gamma_{n_{i}} \delta_{n_{i}}}{2-\alpha_{n_{i}}-\beta_{n_{i}} r+\gamma_{n_{i}} \delta_{n_{i}}-2 \gamma_{n_{i}}}\left\|x_{n_{i}}-\hat{x}\right\|^{2} \\
& +\frac{2 \beta_{n_{i}}}{2-\alpha_{n_{i}}-\beta_{n_{i}} r+\gamma_{n_{i}} \delta_{n_{i}}-2 \gamma_{n_{i}}}\left\langle f(\hat{x})-\hat{x}, j\left(x_{n_{i}+1}-\hat{x}\right)\right\rangle \\
= & \left(1-\frac{2-2 \alpha_{n_{i}}-2 \beta_{n_{i}} r-2 \gamma_{n_{i}} \delta_{n_{i}}}{2-\alpha_{n_{i}}-\beta \beta_{n_{i}} r+\gamma_{n_{i}} \delta_{n_{i}}-2 \gamma_{n_{i}}}\right)\left\|x_{n_{i}}-\hat{x}\right\|^{2} \\
& +\frac{2-2 \alpha_{n_{i}}-2 \beta_{n_{i}} r-2 \gamma_{n_{i}} \delta_{n_{i}}}{2-\alpha_{n_{i}}-\beta_{n_{i}} r+\gamma_{n_{i}} \delta_{n_{i}}-2 \gamma_{n_{i}}} \times \frac{2 \beta_{n_{i}}}{2-2 \alpha_{n_{i}}-2 \beta_{n_{i}} r-2 \gamma_{n_{i}} \delta_{n_{i}}} \\
& \times\left\langle f(\hat{x})-\hat{x}, j\left(x_{n_{i}+1}-\hat{x}\right)\right\rangle .
\end{aligned}
$$

Set $b_{n_{i}}=\frac{2-2 \alpha_{n_{i}}-2 \beta_{n_{i}} r-2 \gamma_{n_{i}}}{\delta_{n_{i}}}$,

$$
c_{n_{i}}=\frac{2-2 \alpha_{n_{i}}-2 \beta_{n_{i}} r-2 \gamma_{n_{i}} \delta_{n_{i}}}{2-\alpha_{n_{i}}-\beta_{n_{i}} r+\gamma{ }_{n_{i}} \delta_{n_{i}}-2 \gamma_{n_{i}}} \times \frac{2 \beta_{n_{i}}}{2-2 \alpha_{n_{i}}-2 \beta_{n_{i}} r-2 \gamma_{n_{i}} \delta_{n_{i}}} .
$$

Then, (3.13) reduces to formula

$$
\left\|x_{n_{i}+1}-\hat{x}\right\|^{2} \leqslant\left(1-b_{n_{i}}\right)\left\|x_{n_{i}}-\hat{x}\right\|^{2}+c_{n_{i}},
$$

and

$$
\begin{aligned}
& b_{n_{i}}=\frac{2 \beta_{n_{i}}(1-r)}{2-\alpha_{n_{i}}-\beta_{n_{i}} r+\gamma_{n_{i}} \delta_{n_{i}}-2 \gamma_{n_{i}}} \\
& =\frac{2 \beta_{n_{i}}(1-r)}{1+\beta_{n_{i}}(1-r)+\gamma_{n_{i}}\left(\delta_{n_{i}}-1\right)} \subset(0,1) \\
& >\frac{2 \beta_{n_{i}}(1-r)}{1+\beta_{n_{i}}(1-r)} \\
& >\beta_{n_{i}}(1-r) \text {. }
\end{aligned}
$$


From condition $\sum_{n=0}^{\infty} \beta_{n}=\infty$, then $\sum_{i=0}^{\infty} b_{n_{i}}=\infty$. Since

$$
\frac{c_{n_{i}}}{b_{n_{i}}}=\frac{2 \beta_{n_{i}}}{2-2 \alpha_{n_{i}}-2 \beta_{n_{i}} r-2 \gamma_{n_{i}} \delta_{n_{i}}}\left\langle f(\hat{x})-\hat{x}, j\left(x_{n_{i}+1}-\hat{x}\right)\right\rangle,
$$

from $\lim _{n \rightarrow \infty} \beta_{n}=0$ and (3.10), we have

$$
\limsup _{n \rightarrow \infty} \frac{c_{n_{i}}}{b_{n_{i}}} \leqslant 0 .
$$

From Lemma 2.5, we obtain that $x_{n_{i}} \rightarrow \hat{x}$ as $i \rightarrow \infty$. The contradiction permits us to conclude that $\left\{x_{n}\right\}$ converges strongly to $\hat{x} \in F(T)$.

Theorem 3.2. Let $\mathrm{X}$ be a real uniformly smooth Banach space and $\mathrm{C}$ be a nonempty bounded closed convex subset of $\mathrm{X}$. Let $\mathrm{N} \geqslant 1$ be an integer and for each $0 \leqslant i \leqslant \mathrm{~N}-1, \mathrm{~T}_{i}: \mathrm{C} \rightarrow \mathrm{C}$ be a $\mu_{i}$-strictly pseudo-contractive mapping such that $\bigcap_{i=0}^{\mathrm{N}-1} \mathrm{~F}\left(\mathrm{~T}_{\mathrm{i}}\right) \neq \emptyset$. Let $\mathrm{f}: \mathrm{C} \rightarrow \mathrm{C}$ a generalized contraction mapping. Pick any $\mathrm{x}_{0} \in \mathrm{C}$. Let $\left\{\mathrm{x}_{\mathrm{n}}\right\}$ be $a$ sequence generated by the following algorithm:

$$
x_{n+1}=\alpha_{n} x_{n}+\beta_{n} f\left(x_{n}\right)+\gamma_{n} S_{n}\left(\delta_{n} x_{n}+\left(1-\delta_{n}\right) x_{n+1}\right),
$$

where $S_{n} x=:\left(1-\lambda_{n}\right) x+\lambda_{n} T_{[n]} x$, and $T_{[n]}=T_{i}$ with $i=n(\bmod N), 0 \leqslant i \leqslant N-1$. Assume that $\left\{\alpha_{n}\right\},\left\{\beta_{n}\right\}$, $\left\{\gamma_{n}\right\},\left\{\delta_{n}\right\}$ and $\left\{\lambda_{n}\right\}$ are five sequences in $[0,1]$ satisfying the following conditions:

(i) $\alpha_{n}+\beta_{n}+\gamma_{n}=1$;

(ii) $\lim _{n \rightarrow \infty}\left|\alpha_{n+1}-\alpha_{n}\right|=0$ and $0<\liminf _{n \rightarrow \infty} \alpha_{n} \leqslant \limsup _{n \rightarrow \infty} \alpha_{n}<1$;

(iii) $\sum_{n=0}^{\infty} \beta_{n}=\infty, \lim _{n \rightarrow \infty} \beta_{n}=0$;

(iv) $0<\delta_{n} \leqslant \delta_{n+1}<1$;

(v) $\lim _{n \rightarrow \infty}\left|\lambda_{n+1}-\lambda_{n}\right|=0$.

Then sequence $\left\{x_{n}\right\}$ converges strongly to a point $\hat{x} \in \bigcap_{i=0}^{N-1} F\left(T_{i}\right)$, which is also the solution of the variational inequality

$$
\langle(I-f) \hat{x}, j(x-\hat{x})\rangle \geqslant 0, \quad \forall x \in \bigcap_{i=0}^{N-1} F\left(T_{i}\right) .
$$

Moreover, assume that $\mathrm{P}$ is the unique sunny nonexpansive retraction from $\mathrm{C}$ onto $\bigcap_{i=0}^{\mathrm{N}-1} \mathrm{~F}\left(\mathrm{~T}_{i}\right)$, the above variational inequality is equivalent to $\mathrm{P} \circ \mathrm{f}(\hat{\mathrm{x}})=\hat{x}$.

Proof. First, we show that $S_{n}$ is nonexpansive, for all $n \geqslant 0$. Indeed, for all $x, y \in C$, taking

$$
0<\varepsilon \leqslant \mu_{i}\left\|\mathrm{~T}_{i} x-\mathrm{T}_{i} \mathrm{y}-(\mathrm{x}-\mathrm{y})\right\|^{2}
$$

for any $0 \leqslant i \leqslant N-1$, by Lemma 2.1 , we have

$$
\begin{aligned}
\left\|S_{n} x-S_{n} y\right\|^{2}= & \left\|\left(1-\lambda_{n}\right) x+\lambda_{n} T_{[n]} x-\left(1-\lambda_{n}\right) y-\lambda_{n} T_{[n]} y\right\|^{2} \\
= & \left\|\left(1-\lambda_{n}\right)(x-y)+\lambda_{n}\left(T_{[n]} x-T_{[n]} y\right)\right\|^{2} \\
\leqslant & 2 \lambda_{n}\left\langle T_{[n]} x-T_{[n]} y, j(x-y)\right\rangle+2 \varepsilon \lambda_{n}+\left(1-2 \lambda_{n}\right)\|x-y\|^{2} \\
\leqslant & 2 \lambda_{n}\left(\|x-y\|^{2}-\mu_{[n]}\left\|T_{[n]} x-T_{[n]} y-(x-y)\right\|^{2}\right) \\
& +\left(1-2 \lambda_{n}\right)\|x-y\|^{2}+2 \varepsilon \lambda_{n} \\
\leqslant & \|x-y\|^{2}-2 \lambda_{n} \mu_{[n]}\left\|T_{[n]} x-T_{[n]} y-(x-y)\right\|^{2}+2 \varepsilon \lambda_{n} \\
\leqslant & \|x-y\|^{2} .
\end{aligned}
$$

It is obvious that for each $n \in N, x \in F\left(S_{n}\right) \Leftrightarrow x \in F\left(T_{[n]}\right)$. So, $F\left(S_{n}\right)=F\left(T_{[n]}\right) \neq \emptyset$. The following proof is the same as that of Theorem 3.1, we can obtain $x_{n} \rightarrow \hat{x} \in F\left(T_{[n]}\right)$, by the uniqueness of convergence of iterative sequence $\left\{x_{n}\right\}$, we have that $x_{n} \rightarrow \hat{x} \in \bigcap_{i=0}^{N-1} F\left(T_{i}\right)$. 
Theorem 3.3. Let $\mathrm{X}$ be a real uniformly smooth Banach space and $\mathrm{C}$ be a nonempty bounded closed convex subset of $\mathrm{X}$. Let $\left\{\mathrm{T}_{\mathfrak{n}}\right\}_{\mathfrak{n}=0}^{\infty}: \mathrm{C} \rightarrow \mathrm{C}$ be a countable family of $\mu$-strictly pseudo-contractive mapping and $\mathrm{f}: \mathrm{C} \rightarrow \mathrm{C}$ a generalized contraction mapping. Suppose that $\left\{\mathrm{T}_{n}\right\}$ satisfies the $\mathrm{PU}$-condition. Let $\mathrm{T}: \mathrm{C} \rightarrow \mathrm{C}$ be a mapping defined by $T x=\lim _{n \rightarrow \infty} T_{n} x$ for all $x \in C$ and suppose that $\mathrm{F}(\mathrm{T})=\bigcap_{\mathrm{n}=0}^{\infty} \mathrm{F}\left(\mathrm{T}_{\mathrm{n}}\right) \neq \emptyset$. Pick any $\mathrm{x}_{0} \in \mathrm{C}$. Let $\left\{x_{n}\right\}$ be a sequence generated by the following algorithm:

$$
x_{n+1}=\alpha_{n} x_{n}+\beta_{n} f\left(x_{n}\right)+\gamma_{n} S_{n}\left(\delta_{n} x_{n}+\left(1-\delta_{n}\right) x_{n+1}\right),
$$

where $S_{n} x=:(1-\lambda) x+\lambda T_{n} x, \lambda \in[0,1]$ and assume that $\left\{\alpha_{n}\right\},\left\{\beta_{n}\right\},\left\{\gamma_{n}\right\}$, and $\left\{\delta_{n}\right\}$ are four sequences in $[0,1]$ satisfying the following conditions:

(i) $\alpha_{n}+\beta_{n}+\gamma_{n}=1$;

(ii) $\lim _{n \rightarrow \infty}\left|\alpha_{n+1}-\alpha_{n}\right|=0$ and $0<\liminf _{n \rightarrow \infty} \alpha_{n} \leqslant \limsup _{n \rightarrow \infty} \alpha_{n}<1$;

(iii) $\sum_{n=0}^{\infty} \beta_{n}=\infty, \lim _{n \rightarrow \infty} \beta_{n}=0$;

(iv) $0<\delta_{n} \leqslant \delta_{n+1}<1$.

Then sequence $\left\{x_{n}\right\}$ converges strongly to a fixed point $\hat{x}$ of $\mathrm{T}$, which is also the solution of the variational inequality

$$
\langle(I-f) \hat{x}, j(y-\hat{x})\rangle \geqslant 0, \quad \forall y \in F(T) .
$$

Moreover, assume that $\mathrm{P}$ is the unique sunny nonexpansive retraction from $\mathrm{C}$ onto $\mathrm{F}(\mathrm{T})=\bigcap_{\mathrm{n}=0}^{\infty} \mathrm{F}\left(\mathrm{T}_{\mathrm{n}}\right)$, then above variational inequality is equivalent to $\mathrm{P} \circ \mathrm{f}(\hat{\mathrm{x}})=\hat{\mathrm{x}}$.

Proof. First, we show that $S_{n}$ is nonexpansive, for all $n \geqslant 0$. Indeed, for all $x, y \in C$, taking

$$
0<\varepsilon \leqslant \mu\left\|T_{n} x-T_{n} y-(x-y)\right\|^{2}
$$

for all $n \geqslant 1$, by Lemma 2.1, we have

$$
\begin{aligned}
\left\|S_{n} x-S_{n} y\right\|^{2}= & \left\|(1-\lambda) x+\lambda T_{n} x-(1-\lambda) y-\lambda T_{n} y\right\|^{2} \\
= & \left\|(1-\lambda)(x-y)+\lambda\left(T_{n} x-T_{n} y\right)\right\|^{2} \\
\leqslant & 2 \lambda\left\langle T_{n} x-T_{n} y, j(x-y)\right\rangle+2 \varepsilon \lambda+(1-2 \lambda)\|x-y\|^{2} \\
\leqslant & 2 \lambda\left(\|x-y\|^{2}-\mu\left\|T_{n} x-T_{n} y-(x-y)\right\|^{2}\right) \\
& +(1-2 \lambda)\|x-y\|^{2}+2 \varepsilon \lambda \\
\leqslant & \|x-y\|^{2}-2 \lambda \mu\left\|T_{n} x-T_{n} y-(x-y)\right\|^{2}+2 \varepsilon \lambda \\
\leqslant & \|x-y\|^{2} .
\end{aligned}
$$

It is obvious that for each $n \in N, x \in F\left(S_{n}\right) \Leftrightarrow x \in F\left(T_{n}\right)$. So, $F\left(S_{n}\right)=F\left(T_{n}\right)$.

Next, we show that $\left\{S_{n}\right\}$ satisfies the PU-condition. From the definition of $S_{n}$ and for all $k, l \in N$, for any subset $B$ of $C$, there exists a continuous and increasing function $h_{B}: R^{+} \rightarrow R^{+}$, we note that

$$
\begin{aligned}
\sup _{\omega \in B} h_{B}\left(\left\|S_{k} \omega-S_{l} \omega\right\|\right) & =\sup _{\omega \in B} h_{B}\left(\lambda\left\|T_{k} \omega-T_{l} \omega\right\|\right) \\
& \leqslant \sup _{\omega \in B} h_{B}\left(\left\|T_{k} \omega-T_{l} \omega\right\|\right) .
\end{aligned}
$$

By our assumption, that $\left\{T_{n}\right\}$ satisfies the PU-condition, by Definition 2.2, we obtain that

$$
\lim _{k, l \rightarrow \infty} \sup _{\omega \in B} h_{B}\left(\left\|S_{k} \omega-S_{l} \omega\right\|\right)=0 .
$$


So, $\left\{S_{n}\right\}$ satisfies the PU-condition. By Lemma 2.3, we can set $S x=\lim _{n \rightarrow \infty} S_{n} x$, for all $x \in C$. It is obvious that $S$ is nonexpansive, and

$$
\begin{aligned}
S x & =\lim _{n \rightarrow \infty} S_{n} x \\
& =\lim _{n \rightarrow \infty}\left[(1-\lambda) x+\lambda T_{n} x\right] \\
& =(1-\lambda) x+\lambda T x .
\end{aligned}
$$

We can observe that $x \in \mathrm{F}(\mathrm{S}) \Leftrightarrow x \in \mathrm{F}(\mathrm{T})$. That is,

$$
F(S)=F(T)=\bigcap_{n=1}^{\infty} F\left(T_{n}\right)=\bigcap_{n=1}^{\infty} F\left(S_{n}\right) \neq \emptyset .
$$

Next, we show that $\lim _{n \rightarrow \infty}\left\|x_{n+1}-x_{n}\right\|=0$.

Set $z_{n}=\frac{x_{n+1}-\alpha_{n} x_{n}}{1-\alpha_{n}}, y_{n}=\delta_{n} x_{n}+\left(1-\delta_{n}\right) x_{n+1}$. We have that

$$
\begin{aligned}
\left\|z_{n+1}-z_{n}\right\| \leqslant & \frac{\beta_{n+1}}{1-\alpha_{n+1}} \psi\left(\left\|x_{n+1}-x_{n}\right\|\right)+\frac{\gamma_{n+1}}{1-\alpha_{n+1}}\left\|S_{n+1} y_{n+1}-S_{n} y_{n}\right\| \\
& +\left|\frac{\beta_{n+1}}{1-\alpha_{n+1}}-\frac{\beta_{n}}{1-\alpha_{n}}\right|\left\|f\left(x_{n}\right)-S_{n} y_{n}\right\| .
\end{aligned}
$$

Since

and

$$
\begin{aligned}
\left\|S_{n+1} y_{n+1}-S_{n} y_{n}\right\|= & \left\|S_{n+1} y_{n+1}-S_{n+1} y_{n}+S_{n+1} y_{n}-S_{n} y_{n}\right\| \\
\leqslant & \left\|y_{n+1}-y_{n}\right\|+\left\|S_{n+1} y_{n}-S_{n} y_{n}\right\| \\
\leqslant & \left(1-\delta_{n+1}\right)\left\|x_{n+2}-x_{n+1}\right\|+\delta_{n}\left\|x_{n+1}-x_{n}\right\| \\
& +\left\|S_{n+1} y_{n}-S_{n} y_{n}\right\|
\end{aligned}
$$

$$
\begin{aligned}
\left\|x_{n+2}-x_{n+1}\right\| \leqslant & \alpha_{n+1}\left\|x_{n+1}-x_{n}\right\|+\beta_{n+1} \psi\left(\left\|x_{n+1}-x_{n}\right\|\right) \\
& +\left|\alpha_{n+1}-\alpha_{n}\right|\left\|x_{n}-S_{n} y_{n}\right\|+\left|\beta_{n+1}-\beta_{n}\right|\left\|f\left(x_{n}\right)-S_{n} y_{n}\right\| \\
& +\gamma_{n+1}\left\|S_{n+1} y_{n+1}-S_{n} y_{n}\right\|,
\end{aligned}
$$

substituting (3.17) into (3.18), we obtain that

$$
\begin{aligned}
\left\|x_{n+2}-x_{n+1}\right\| \leqslant & \frac{\alpha_{n+1}+\gamma_{n+1} \delta_{n}+\beta_{n+1} \psi}{1-\gamma_{n+1}\left(1-\delta_{n+1}\right)}\left\|x_{n+1}-x_{n}\right\| \\
& +\frac{\left|\alpha_{n+1}-\alpha_{n}\right|}{1-\gamma_{n+1}\left(1-\delta_{n+1}\right)}\left\|x_{n}-S_{n} y_{n}\right\| \\
& +\frac{\left|\beta_{n+1}-\beta_{n}\right|}{1-\gamma_{n+1}\left(1-\delta_{n+1}\right)}\left\|f\left(x_{n}\right)-S_{n} y_{n}\right\| \\
& +\frac{\gamma_{n+1}}{1-\gamma_{n+1}\left(1-\delta_{n+1}\right)}\left\|S_{n+1} y_{n}-S_{n} y_{n}\right\| .
\end{aligned}
$$

Substituting (3.19) and (3.17) into (3.16), we obtain that

$$
\begin{aligned}
\left\|z_{n+1}-z_{n}\right\| \leqslant & \left(1-\frac{\beta_{n+1} \eta}{1-\alpha_{n+1}}\right)\left\|x_{n+1}-x_{n}\right\|+\frac{\left|\alpha_{n+1}-\alpha_{n}\right|}{1-\gamma_{n+1}\left(1-\delta_{n+1}\right)}\left\|x_{n}-S_{n} y_{n}\right\| \\
& +\left[\frac{\left|\beta_{n+1}-\beta_{n}\right|}{1-\gamma_{n+1}\left(1-\delta_{n+1}\right)}+\left|\frac{\beta_{n+1}}{1-\alpha_{n+1}}-\frac{\beta_{n}}{1-\alpha_{n}}\right|\right]\left\|f\left(x_{n}\right)-S_{n} y_{n}\right\| \\
& +\left[\frac{\gamma_{n+1}}{1-\gamma_{n+1}\left(1-\delta_{n+1}\right)}+\frac{\gamma_{n+1}}{1-\alpha_{n+1}}\right]\left\|S_{n+1} y_{n}-S_{n} y_{n}\right\| .
\end{aligned}
$$


We know that

$$
\frac{1}{2}\left\|S_{n+1} y_{n}-S_{n} y_{n}\right\| \leqslant \frac{1}{2}\left\|S_{n+1} y_{n}-S y_{n}\right\|+\frac{1}{2}\left\|S y_{n}-S_{n} y_{n}\right\|
$$

From $\left\{x_{n}\right\},\left\{y_{n}\right\} \subset C$, assume that there exists a subset $B$ of $C$ which contains $\left\{x_{n}\right\},\left\{y_{n}\right\}$. Since $h_{B}: R^{+} \rightarrow$ $\mathrm{R}^{+}$is a continuous, increasing and convex function, then we have

$$
\begin{aligned}
h_{B}\left(\frac{1}{2}\left\|S_{n+1} y_{n}-S_{n} y_{n}\right\|\right) \leqslant & \frac{1}{2} h_{B}\left(\left\|S_{n+1} y_{n}-S y_{n}\right\|\right)+\frac{1}{2} h_{B}\left(\left\|S y_{n}-S_{n} y_{n}\right\|\right) \\
\leqslant & \frac{1}{2} \sup _{\omega \in B} h_{B}\left(\left\|S_{n+1} \omega-S \omega\right\|\right) \\
& +\frac{1}{2} \sup _{\omega \in B} h_{B}\left(\left\|S \omega-S_{n} \omega\right\|\right) .
\end{aligned}
$$

By Lemma 2.3 and the continuity of $h_{B}$, we obtain that $\lim _{n \rightarrow \infty} h_{B}\left(\frac{1}{2}\left\|S_{n+1} y_{n}-S_{n} y_{n}\right\|\right)=0$. This implies that

$$
\lim _{n \rightarrow \infty}\left\|S_{n+1} y_{n}-S_{n} y_{n}\right\|=0
$$

Since $C$ is a nonempty bounded closed convex subset of $X$, then $\left\{x_{n}\right\},\left\{y_{n}\right\},\left\{f\left(x_{n}\right)\right\}$ and $\left\{S_{n} y_{n}\right\}$ are bounded. Hence $\left\|x_{n}-S_{n} y_{n}\right\|$ and $\left\|f\left(x_{n}\right)-S_{n} y_{n}\right\|$ are bounded. Again from conditions (ii) and (iii), we have

$$
\limsup _{n \rightarrow \infty}\left(\left\|z_{n+1}-z_{n}\right\|-\left\|x_{n+1}-x_{n}\right\|\right) \leqslant 0 .
$$

By using Lemma 2.4, we have

$$
\lim _{n \rightarrow \infty}\left\|z_{n}-x_{n}\right\|=0
$$

Since

$$
\lim _{n \rightarrow \infty}\left\|x_{n+1}-x_{n}\right\|=\lim _{n \rightarrow \infty}\left(1-\alpha_{n}\right)\left\|z_{n}-x_{n}\right\|=0 .
$$

Next, we prove that $\lim _{n \rightarrow \infty}\left\|x_{n}-S x_{n}\right\|=0$.

We observe that

$$
\begin{aligned}
\frac{1}{2}\left\|x_{n}-S x_{n}\right\| & \leqslant \frac{1}{2}\left\|x_{n}-S_{n} x_{n}\right\|+\frac{1}{2}\left\|S_{n} x_{n}-S x_{n}\right\| \\
& \leqslant \frac{1}{2}\left\|x_{n}-S_{n} x_{n}\right\|+\frac{1}{2} \sup _{\omega \in B}\left\|S_{n} \omega-S \omega\right\| .
\end{aligned}
$$

On the other hand, we have that

$$
\lim _{n \rightarrow \infty}\left\|x_{n}-S_{n} x_{n}\right\|=0
$$

This proof is the same as that of Theorem 3.1.

Since $h_{B}: R^{+} \rightarrow R^{+}$is continuous, increasing and convex, then we have

$$
\begin{aligned}
h_{B}\left(\frac{1}{2}\left\|x_{n}-S x_{n}\right\|\right) & \leqslant \frac{1}{2} h_{B}\left(\left\|x_{n}-S_{n} x_{n}\right\|\right)+\frac{1}{2} h_{B}\left(\left\|S_{n} x_{n}-S x_{n}\right\|\right) \\
& \leqslant \frac{1}{2} h_{B}\left(\left\|x_{n}-S_{n} x_{n}\right\|\right)+\frac{1}{2} \sup _{\omega \in B} h_{B}\left(\left\|S_{n} \omega-S \omega\right\|\right) .
\end{aligned}
$$

From Lemma 2.3 and the continuity of $h_{B}$, we obtain that $\lim _{n \rightarrow \infty} h_{B}\left(\frac{1}{2}\left\|x_{n}-S x_{n}\right\|\right)=0$. This implies that

$$
\lim _{n \rightarrow \infty}\left\|x_{n}-S x_{n}\right\|=0 .
$$

This remaining proof is the same as that of Theorem 3.1, we omit it. Therefore, we conclude that $\left\{x_{n}\right\}$ converges strongly to $\hat{x} \in \bigcap_{n=1}^{\infty} F\left(T_{n}\right)$. 


\section{Numerical experiments}

In this section, respectively, we give the corresponding numerical examples of Theorem 3.1, Theorem 3.2 and Theorem 3.3 to illustrate the effectiveness of the three algorithms constructed in Section 3. All codes were written in Matlab 2010b and run on Dell i - 5 Dual-Core laptop.

Example 4.1. Let $X:=R$ and $C:=[0,1] \subset R$. Let $T: C \rightarrow C, f: C \rightarrow C$ be defined by

$$
T(x)=\frac{1}{4} x, \quad f(x)=\frac{1}{2} x .
$$

It is clear that $\mathrm{F}(\mathrm{T})=\{0\}$.

Let us choose

$$
\alpha_{n}=\frac{1}{2}-\frac{1}{4 n}, \quad \beta_{n}=\frac{1}{4 n}, \quad \gamma_{n}=\frac{1}{2}, \quad \delta_{n}=\frac{n}{2(n+1)}, \quad \lambda_{n}=\frac{1}{3}-\frac{1}{4 n} .
$$

Obviously, $X, C, T, f, \alpha_{n}, \beta_{n}, \gamma_{n}, \delta_{n}, \lambda_{n}$ satisfy all the conditions of Theorem 3.1.

Below we take different initial values to observe the convergence of the algorithm (3.1). Taking $x_{0}=$ $0.1, x_{0}=0.5, x_{0}=1$, respectively, we have the following numerical results:

Table 1: numerical examples of Theorem 3.1.

\begin{tabular}{lccccccccc}
\hline Iter. & $\mathrm{n}$ & 0 & 5 & 10 & 15 & 20 & 25 & 30 & $\ldots$ \\
$x_{0}=0.1000$ & $x_{n}$ & 0.1000 & 0.0342 & 0.0139 & 0.0058 & 0.0025 & 0.0010 & $4.4419 \times 10^{-4}$ & $\ldots$ \\
$x_{0}=0.5000$ & $x_{n}$ & 0.5000 & 0.1711 & 0.0697 & 0.0291 & 0.0123 & 0.0052 & 0.0022 & $\ldots$ \\
$x_{0}=1.0000$ & $x_{n}$ & 1.0000 & 0.3422 & 0.1393 & 0.0582 & 0.0245 & 0.0104 & 0.0044 & $\ldots$ \\
\hline
\end{tabular}

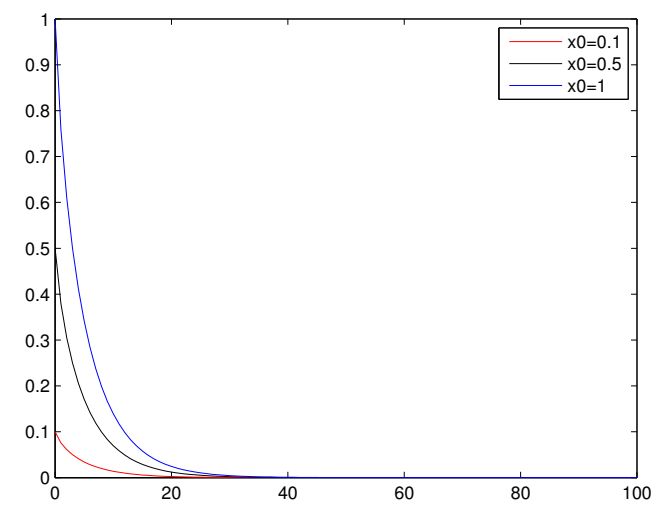

Figure 1: numerical examples of Theorem 3.1.

Example 4.2. Let $X, C, f, \alpha_{n}, \beta_{n}, \gamma_{n}, \delta_{n}, \lambda_{n}$ is the same to that of Example 4.1 and $T_{i}: C \rightarrow C$ be defined as follows:

$$
T_{i}(x)= \begin{cases}\frac{1}{N} x, & i=0, \\ \frac{1}{i} x, & 1 \leqslant i \leqslant N-1 .\end{cases}
$$

Obviously, $\bigcap_{i=0}^{N-1} F\left(T_{i}\right)=\{0\}$ and $X, C, T_{i}, f, \alpha_{n}, \beta_{n}, \gamma_{n}, \delta_{n}, \lambda_{n}$ satisfy all the conditions of Theorem 3.2.

Below we consider two situations to observe the convergence of the algorithm (3.14).

Case 1. Taking $N=10, x_{0}=0.1, x_{0}=0.5, x_{0}=1$, respectively, we have the following numerical results: 
Table 2: numerical examples of Theorem 3.2 for $\mathrm{N}=10$.

\begin{tabular}{lccccccccc}
\hline Iter. & $\mathrm{n}$ & 0 & 5 & 10 & 15 & 20 & 25 & 30 & $\ldots$ \\
$x_{0}=0.1000$ & $x_{n}$ & 0.1000 & 0.0377 & 0.0135 & 0.0070 & 0.0026 & 0.0014 & $5.1472 \times 10^{-4}$ & $\ldots$ \\
$x_{0}=0.5000$ & $x_{n}$ & 0.5000 & 0.1886 & 0.0676 & 0.0352 & 0.0130 & 0.0069 & 0.0026 & $\ldots$ \\
$x_{0}=1.0000$ & $x_{n}$ & 1.0000 & 0.3771 & 0.1353 & 0.0704 & 0.0261 & 0.0139 & 0.0052 & $\ldots$ \\
\hline
\end{tabular}

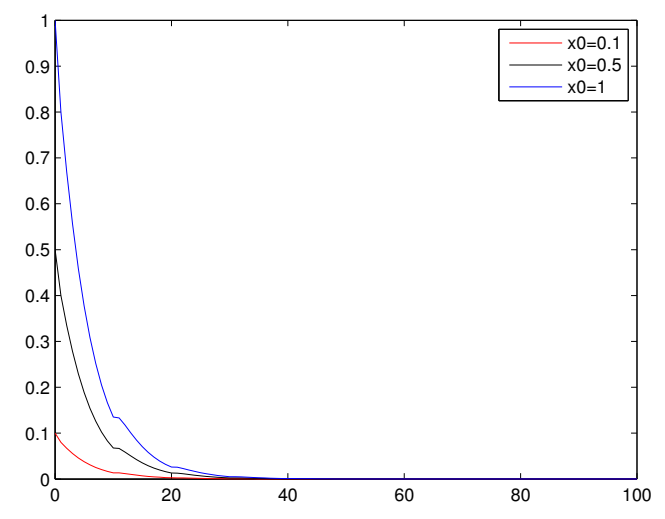

Figure 2: numerical examples of Theorem 3.2 for $\mathrm{N}=10$.

Case 2. Taking $x_{0}=1, N=5, N=10, N=20$, respectively, we have the following numerical results:

Table 3: numerical examples of Theorem 3.2 for $x_{0}=1$.

\begin{tabular}{lccccccccc}
\hline Iter. & $\mathrm{n}$ & 0 & 5 & 10 & 15 & 20 & 25 & 30 & $\ldots$ \\
$\mathrm{N}=5$ & $x_{\mathrm{n}}$ & 1.0000 & 0.3771 & 0.1899 & 0.0989 & 0.0522 & 0.0278 & 0.0148 & $\ldots$ \\
$\mathrm{N}=10$ & $x_{\mathrm{n}}$ & 1.0000 & 0.3771 & 0.1353 & 0.0704 & 0.0261 & 0.0139 & 0.0052 & $\ldots$ \\
$\mathrm{N}=20$ & $x_{\mathrm{n}}$ & 1.0000 & 0.3771 & 0.1353 & 0.0469 & 0.0160 & 0.0085 & 0.0032 & $\ldots$ \\
\hline
\end{tabular}

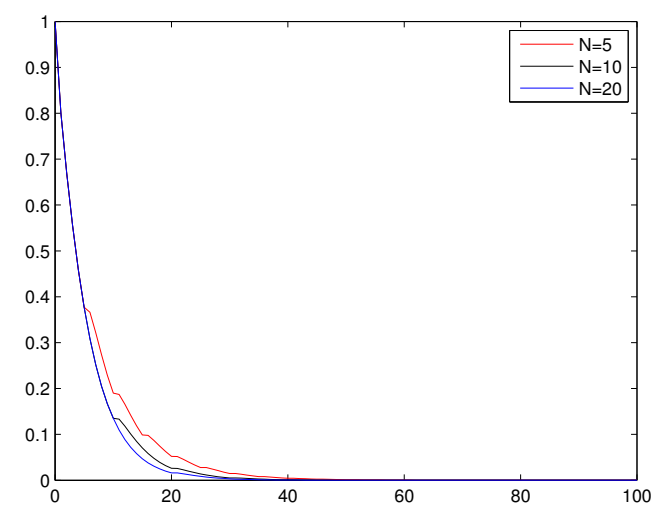

Figure 3: numerical examples of Theorem 3.2 for $x_{0}=1$.

Example 4.3. Let $X, C, f, \alpha_{n}, \beta_{n}, \gamma_{n}, \delta_{n}$ is the same to that of Example 4.1 and $T_{n}: C \rightarrow C$ be defined by $T_{n}(x)=\frac{1}{n} x$. Obviously, $\bigcap_{n=1}^{\infty} F\left(T_{n}\right)=\{0\}$ and $X, C, T, f, \alpha_{n}, \beta_{n}, \gamma_{n}, \delta_{n}$ satisfy all the conditions of 


\section{Theorem 3.3.}

Below we also consider two situations to observe the convergence of the algorithm (3.15).

Case 1. Taking $\lambda=\frac{2}{3}, x_{0}=0.1, x_{0}=0.5, x_{0}=1$, respectively, we have the following numerical results:

Table 4: numerical examples of Theorem 3.3 for $\lambda=\frac{2}{3}$.

\begin{tabular}{|c|c|c|c|c|c|c|c|c|c|}
\hline Iter. & $\mathrm{n}$ & 0 & 5 & 10 & 15 & 20 & 25 & 30 & 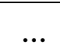 \\
\hline$x_{0}=0.1000$ & $x_{n}$ & 0.1000 & 0.0170 & 0.0020 & $2.2809 \times 10^{-4}$ & $2.5175 \times 10^{-5}$ & $2.7449 \times 10^{-6}$ & $4.6350 \times 10^{-7}$ & .. \\
\hline$x_{0}=0.5000$ & $x_{n}$ & 0.5000 & 0.0851 & 0.0101 & 0.0011 & $1.2588 \times 10^{-4}$ & $1.3724 \times 10^{-5}$ & $1.4847 \times 10^{-6}$ & .. \\
\hline$x_{0}=1.0000$ & $x_{n}$ & 1.0000 & 0.1702 & 0.0202 & 0.0023 & $2.5175 \times 10^{-4}$ & $2.7449 \times 10^{-5}$ & $2.9693 \times 10^{-6}$ & .. \\
\hline
\end{tabular}

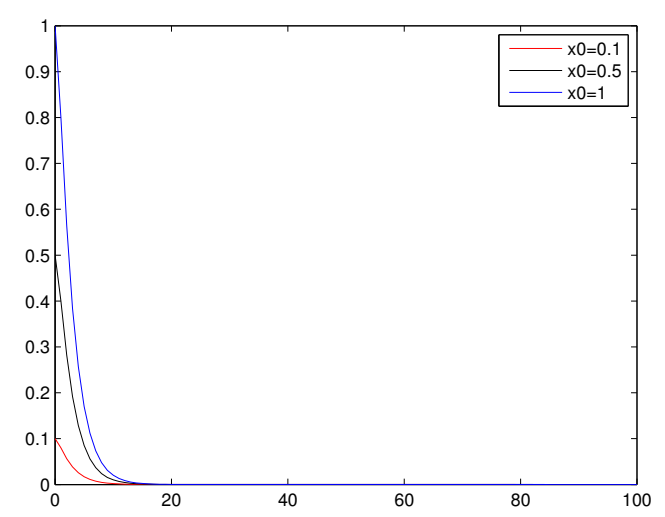

Figure 4: numerical examples of Theorem 3.3 for $\lambda=\frac{2}{3}$.

Case 2. Taking $x_{0}=1, \lambda=\frac{1}{2}, \lambda=\frac{1}{4}, \lambda=\frac{2}{3}$, respectively, we have the following numerical results:

Table 5: numerical examples of Theorem 3.3 for $x_{0}=1$.

\begin{tabular}{|c|c|c|c|c|c|c|c|c|c|}
\hline Iter. & $\mathrm{n}$ & 0 & 5 & 10 & 15 & 20 & 25 & 30 & $\ldots$ \\
\hline$\lambda=\frac{1}{2}$ & $x_{n}$ & 1.0000 & 0.2350 & 0.0463 & 0.0089 & 0.0017 & $3.2123 \times 10^{-4}$ & $6.0659 \times 10^{-5}$ & $\ldots$ \\
\hline$\lambda=\frac{1}{4}$ & $x_{n}$ & 1.0000 & 0.3825 & 0.1601 & 0.0680 & 0.0290 & 0.0124 & 0.0053 & $\ldots$ \\
\hline$\lambda=\frac{2}{3}$ & $x_{n}$ & 1.0000 & 0.1702 & 0.0202 & 0.0023 & $2.5175 \times 10^{-4}$ & $2.7449 \times 10^{-5}$ & $2.9693 \times 10^{-6}$ & $\cdots$ \\
\hline
\end{tabular}

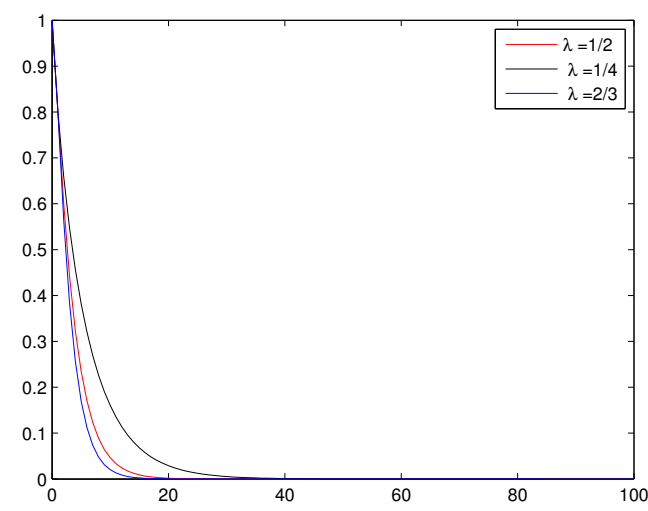

Figure 5: numerical examples of Theorem 3.3 for $x_{0}=1$. 


\section{Acknowledgment}

The authors are most grateful to the referees and the editor for their helpful comments and advices which improved the contents of this paper.

\section{References}

[1] M. A. Alghamdi, M. A. Alghamdi, N. Shahzad, H.K. Xu, The implicit midpoint rule for nonexpansive mappings, Fixed Point Theory Appl., 2014 (2014), 9 pages. 1

[2] G. Bader, P. Deuhard, A semi-implicit mid-point rule for stiff systems of ordinary differential equations, Numer. Math., 41 (1983), 373-398. 1

[3] R. E. Bruck, Nonexpansive retracts of Banach spaces, Bull. Amer. Math. Soc., 76 (1970), 384-386. 2

[4] P. Deuhard, Recent progress in extrapolation methods for ordinary differential equations, SIAM Rev., 27 (1985), 505-535. 1

[5] Q.-W. Fan, X.-Y. Wang, An explicit iterative algorithm for $k$-strictly pseudo-contractive mappings in Banach spaces, J. Nonlinear Sci. Appl., 9 (2016), 5021-5028. 1

[6] Y. Ke, C. Ma, The generalized viscosity implicit rules of nonexpansive mappings in Hilbert spaces, Fixed Point Theory Appl., 2015 (2015), 21 pages. 1, 1, 1

[7] T. C. Lim, On characterizations of Meir-Keeler contractive maps, Nonlinear Anal., 46 (2001), 113-120. 1.1

[8] A. Moudafi, Viscosity approximation methods for fixed-points problems, J. Math. Anal. Appl., 241 (2000), 46-55. 1

[9] J. A. Park, Mann-iteration process for the fixed point of strictly pseudocontractive mapping in some Banach spaces, J. Korean Math. Soc., 31 (1994), 333-337. 2.1

[10] S. Plubtieng, K. Ungchittrakool, Approximation of common fixed points for a countable family of relatively nonexpansive mappings in a Banach space and applications, Nonlinear Anal., 72 (2010), 2896-2908. 2.2, 2.3

[11] S. Reich, Asymptotic behavior of contractions in Banach spaces, J. Math. Anal. Appl., 44 (1973), 57-70. 2, 2.6

[12] S. Somali, Implicit midpoint rule to the nonlinear degenerate boundary value problems, Int. J. Comput. Math., 79 (2002), 327-332. 1

[13] P. Sunthrayuth, P. Kumam, Viscosity approximation methods based on generalized contraction mappings for a countable family of strict pseudo-contractions, a general system of variational inequalities and a generalized mixed equilibrium problem in Banach spaces, Math. Comput. Modell., 58 (2013), 1814-1828. 2.8, 2.9

[14] T. Suzuki, Strong convergence of Krasnoselskii and Mann's type sequences for one-parameter nonexpansive semi-groups without Bochner integrals, J. Math. Anal. Appl., 305 (2005), 227-239. 2.4

[15] T. Suzuki, Moudafi's viscosity approximations with Meir-Keeler contractions, J. Math. Anal. Appl., 325 (2007), 342-352. $1.2,2.7$

[16] W. Takahashi, Nonlinear Functional Analysis, Yokohama Publishers, Yokohama, (2000). 2

[17] H.-K. Xu, Viscosity approximation methods for nonexpansive mappings, J. Math. Anal. Appl., 298 (2004), 279-291. 1, 2.5

[18] H.-K. Xu, M. A. Alghamdi, N. Shahzad, The viscosity technique for the implicit midpoint rule of nonexpansive mappings in Hilbert spaces, Fixed Point Theory Appl., 2015 (2015), 12 pages. 1, 1

[19] Q. Yan, G. Cai, P. Luo, Strong convergence theorems for the generalized viscosity implicit rules of nonexpansive mappings in uniformly smooth Banach spaces, J. Nonlinear Sci. Appl., 9 (2016), 4039-4051. 1

[20] Y. Yao, N. Shahzad, Y. C. Liou, Modified semi-implicit midpoint rule for nonexpansive mappings, Fixed Point Theory Appl., 2015 (2015), 15 pages. 1 